\title{
Local and regional recurrence following mastectomy in breast cancer patients with 1-3 positive nodes: implications for postmastectomy radiotherapy volume
}

\author{
Shin-Hyung Park, MD, PhD', Jeeyeon Lee, MD, PhD², Jeong Eun Lee, MD, PhD', \\ Min Kyu Kang, MD1, Mi Young Kim, MD², Ho Yong Park, MD, PhD², Jin Hyang Jung, MD, PhD², \\ Yee Soo Chae, MD, PhD ${ }^{4}$, Soo Jung Lee, MD, PhD ${ }^{4}$, Jae-Chul Kim, MD, PhD ${ }^{1}$ \\ ${ }^{1}$ Department of Radiation Oncology, School of Medicine, Kyungpook National University, Daegu; \\ ${ }^{2}$ Department of Surgery, School of Medicine, Kyungpook National University, Daegu; \\ ${ }^{3}$ Department of Radiation Oncology, Kyungpook National University Chilgok Hospital, Daegu; \\ ${ }^{4}$ Deaprtment of Hemato-Oncology, School of Medicine, Kyungpook National University, Daegu, Korea
}

\begin{abstract}
Purpose: To determine the necessity of postmastectomy radiotherapy (PMRT) and which regions would be at risk for recurrence, we evaluated local and regional recurrence in breast cancer patients with 1-3 positive nodes and a tumor size of $<5 \mathrm{~cm}$.

Materials and Methods: We retrospectively analyzed data of 133 female breast cancer patients with 1-3 positive nodes, and a tumor size of $<5 \mathrm{~cm}$ who were treated with mastectomy followed by adjuvant systemic therapy between 2007 and 2016. The median follow-up period was 57 months (range, 12 to 115 months). Most patients (82.7\%) were treated with axillary lymph node dissection. Adjuvant chemotherapy, endocrine therapy, and trastuzumab therapy were administered to 124 patients (93.2\%), $112(84.2 \%)$, and $33(24.8 \%)$, respectively. The most common chemotherapy regimen was anthracycline and cyclophosphamide followed by taxane (71.4\%).

Results: Three patients (2.3\%), 8 (6.0\%), and 12 (9.0\%) experienced local, regional, and distant failures, respectively. The 5-year cumulative risk of local recurrence, regional recurrence, distant metastasis, and disease-free survival was $3.1 \%, 8.0 \%, 11.7 \%$, and 83.4\%, respectively. There were no statistically significant clinicopathologic factors associated with local recurrence. Lymphovascular invasion (univariate $p=0.015$ and multivariate $p=0.054$ ) was associated with an increased risk of regional recurrence.

Conclusion: Our study showed a very low local recurrence in patients with 1-3 positive nodes and tumor size of $<5 \mathrm{~cm}$ who were treated with mastectomy and modern adjuvant systemic treatment. The PMRT volume need to be tailored for each patient's given risk for local and regional recurrence, and possible radiation-related toxicities.
\end{abstract}

Keywords: Breast neoplasms, Recurrence, Mastectomy, Radiotherapy

\section{Introduction}

Postmastectomy radiotherapy (PMRT) is an important component of breast cancer treatment when microscopic residual disease may be present in the chest wall or regional lymph nodes, despite previous mastectomy and/or adjuvant

Received 08 October 2018, Revised 11 November 2018, Accepted 28 November 2018.

Correspondence: Jae-Chul Kim, MD, PhD, Department of Radiation Oncology, School of Medicine, Kyungpook National University, 130 Dongdeok-ro, Jung-gu, Daegu 41944, Korea. Tel: +82-53-200-5352, Fax: +82-53-426-3303, E-mail: jckim@knu. ac.kr (http://orcid.org/0000-0002-7553-3552)

(c) This is an Open Access article distributed under the terms of the Creative Commons Attribution Non-Commercial License (http://creativecommons.org/ licenses/by-nc/4.0/) which permits unrestricted non-commercial use, distribution, and reproduction in any medium, provided the original work is properly cited.

www.e-roj.org 
systemic therapy. Microscopic residual disease can cause locoregional recurrence and distant metastases. Therefore, PMRT is used to eradicate microscopic residual cancer cells and lower the risk of locoregional recurrence and distant metastasis, which enhances survival.

Randomized trials have suggested that PMRT has a beneficial effect on survival, as well as on locoregional recurrence [1-3]. For patients with $\geq 4$ positive lymph nodes, PMRT is common practice at many institutions. However, the application of these trial data on patients with 1-3 positive nodes remains controversial [4]. Results from the Danish $82 \mathrm{~B} / \mathrm{C}$ trials [3] and the Vancouver British Columbia trial [2] suggested that patients with 1-3 positive lymph nodes benefited from PMRT in terms of locoregional recurrence and prolonged survival. In addition, the newly updated data in the Early Breast Cancer Trialists' Collaborative Group (EBCTCG) meta-analysis from randomized trials demonstrated the benefit of PMRT in these patients [5]. Consequently, the National Comprehensive Cancer Network (NCCN) guidelines [6] strongly recommend PMRT to the ipsilateral chest wall and regional lymphatic area, even in patients with 1-3 positive nodes following mastectomy. However, more recent studies [710] reported a lower locoregional recurrence risk than those from historical trials $[2,3,5]$ for patients with 1-3 positive nodes who were treated with mastectomy and modern adjuvant systemic therapy.

Moreover, PMRT volumes are also controversial. These usually include the ipsilateral chest wall with or without the regional nodal area. However, not all PMRT-indicated patients have the same risk for local and regional recurrence. Additionally, applying chest wall irradiation, regional nodal irradiation, or both could affect treatment efficacy and complications. To design an adequate radiotherapy plan, specific knowledge regarding failure patterns is necessary.

This study aimed to determine the necessity of PMRT and the regions that would be considered at risk for recurrence, by retrospectively evaluating local and regional recurrence risks in patients treated with mastectomy and modern adjuvant systemic treatment.

\section{Materials and Methods}

We performed a retrospective analysis of 436 consecutive patients who had pathologically proven breast cancer with a $<5 \mathrm{~cm}$ tumor and 1-3 positive axillary lymph nodes at our institution between 2007 and 2016. This study was approved by Kyungpook National University Chilgok Hospital Institutional
Review Board (No. 2018-03-018). Of 436 patients, 205 and 231 were treated with mastectomy and breast-conserving surgery, respectively. From the 205 patients treated with mastectomy, we excluded patients who received neoadjuvant chemotherapy before mastectomy (58 patients), received adjuvant radiotherapy (9 patients), or were followed-up for a short duration ( $<3$ months) after mastectomy (5 patients). A total of 133 patients were included in the analysis. The expression of estrogen receptor (ER), progesterone receptor (PR), human epidermal growth factor receptor 2 (HER2), and Ki67 was scored based on the CAP/ASCO guidelines [11,12], and each breast cancer was categorized as a molecular subtype based on the expression of these four proteins (luminal A, B, HER2 positive, and basal type), as described in the St. Gallen guidelines [13].

The primary endpoint of this study was to evaluate the risk of local and regional recurrence. The secondary endpoints were to evaluate distant metastasis risk and disease-free survival. Local recurrence was defined as an ipsilateral chest wall recurrence. Regional recurrence was defined as recurrence of the ipsilateral axillary lymph node, internal mammary lymph node, or the supraclavicular lymph node. Distant metastasis was defined as metastasis to distant sites. Disease-free survival was defined as any disease recurrence or any cause of death. All endpoints were calculated from the date of mastectomy and were estimated using the Kaplan-Meier method. Statistical differences were compared using the log-rank test in a univariate analysis. Multivariate analysis using the Cox proportional hazard modeling was performed to identify independent predictors among the prognostic factors. The $p$-values of $<0.05$ were considered statistically significant. All statistical analyses were performed using R statistical software version 3.4.3 (The R Project, http://www.R-project.org).

\section{Results}

Table 1 summarizes the patient and tumor-related characteristics. Median age was 49 years (range, 23 to 76

Table 1. Patient and tumor characteristics $(n=133)$

\begin{tabular}{lc}
\hline \multicolumn{1}{c}{ Characteristic } & Value \\
\hline Age $(\mathrm{yr})$ & $49(23-76)$ \\
$\leq 40$ & $25(18.8)$ \\
$>40$ & $108(81.2)$ \\
Pathology & \\
Invasive ductal carcinoma & $119(89.5)$ \\
Invasive lobular carcinoma & $11(8.3)$ \\
Others & $3(2.3)$ \\
\hline
\end{tabular}

Continued on the next page.

www.e-roj.org

286 
Table 1. Continued

\begin{tabular}{|c|c|}
\hline Characteristic & Value \\
\hline \multicolumn{2}{|l|}{ Tumor size } \\
\hline$\leq 2 \mathrm{~cm}$ & $56(42.1)$ \\
\hline$>2 \mathrm{~cm}$ & 77 (57.9) \\
\hline \multicolumn{2}{|l|}{ Histologic grade } \\
\hline 1 & $18(13.5)$ \\
\hline 2 & 81 (60.9) \\
\hline 3 & $34(25.6)$ \\
\hline \multicolumn{2}{|l|}{ Nuclear grade } \\
\hline 1 & $6(4.5)$ \\
\hline 2 & $60(45.1)$ \\
\hline 3 & 65 (48.9) \\
\hline Unknown & $2(1.5)$ \\
\hline \multicolumn{2}{|l|}{ Multiplicity } \\
\hline Yes & $40(30.1)$ \\
\hline No & $93(69.9)$ \\
\hline \multicolumn{2}{|l|}{ Estrogen receptor status ${ }^{\mathrm{a})}$} \\
\hline Positive & 105 (78.9) \\
\hline Negative & $28(21.1)$ \\
\hline \multicolumn{2}{|c|}{ Progesterone receptor status ${ }^{\mathrm{a})}$} \\
\hline Positive & $87(65.4)$ \\
\hline Negative & $46(34.6)$ \\
\hline \multicolumn{2}{|l|}{ HER2 status } \\
\hline Positive $^{\text {b) }}$ & $35(26.3)$ \\
\hline Negative & $98(73.7)$ \\
\hline \multicolumn{2}{|l|}{ Ki-67 } \\
\hline$<14 \%$ & $66(49.6)$ \\
\hline$\geq 14 \%$ & 53 (39.8) \\
\hline Unknown & $14(10.5)$ \\
\hline \multicolumn{2}{|l|}{ Molecular subtype } \\
\hline Luminal A & $64(48.1)$ \\
\hline Luminal B & $42(31.6)$ \\
\hline HER2 & $12(9.0)$ \\
\hline Basal & $15(11.3)$ \\
\hline \multicolumn{2}{|l|}{ Lymphovascular invasion } \\
\hline Present & $63(47.4)$ \\
\hline None & 69 (51.9) \\
\hline Unknown & $1(0.8)$ \\
\hline \multicolumn{2}{|l|}{ Extracapsular extension } \\
\hline Present & $6(4.5)$ \\
\hline None & $126(94.7)$ \\
\hline Unknown & $1(0.8)$ \\
\hline \multicolumn{2}{|l|}{ No. of positive lymph nodes } \\
\hline 1 & $60(45.1)$ \\
\hline 2 & $52(39.1)$ \\
\hline 3 & $21(15.8)$ \\
\hline \multicolumn{2}{|c|}{ No. of dissected lymph nodes } \\
\hline$<10$ & $38(28.6)$ \\
\hline$\geq 10$ & $95(71.4)$ \\
\hline
\end{tabular}

Values are presented as median (range) or number (\%).

${ }^{a}$ Determined by immunohistochemical stain of estrogen receptor and progesterone receptor.

${ }^{b)}$ Determined by immunohistochemical stain $(3+)$ or silver in situ hybridization (+). years). Tumor size was $\leq 2 \mathrm{~cm}$ in 56 patients (42.1\%) and $2-5$ $\mathrm{cm}$ in 77 patients (57.9\%). Lymphovascular invasion and extracapsular extension were observed in 63 patients (47.4\%) and 6 patients (4.5\%), respectively. The number of positive lymph nodes were 1, 2, and 3 in 60 patients (45.1\%), 52 (39.1\%) and 21 (15.8\%), respectively. Molecular subtypes were luminal A, B, HER2, and basal type in 64 patients (48.1\%), 42 (31.6\%), 12 (9.0\%), and 15 (11.3\%), respectively. Median follow-up period was 57 months (range, 12 to 115 months).

Table 2 summarizes patient treatment-related characteristics. One-hundred and ten patients (82.7\%) were treated with axillary lymph nodes dissection and 23 patients (17.3\%) were treated with sentinel lymph node biopsy alone. Adjuvant chemotherapy, endocrine therapy, and trastuzumab therapy were given to 124 patients (93.2\%), 112 (84.2\%), and 33 (24.8\%), respectively. The most common chemotherapy regimen was anthracycline and cyclophosphamide, followed by taxane (95 patients, 71.4\%).

\section{Local and regional recurrence}

Overall, 3 patients (2.3\%) developed local recurrence. Of these, 2 patients had isolated local recurrence and 1 patient had concurrent regional recurrence and distant metastasis with local recurrence. One patient who had an isolated local recurrence later developed distant metastases at 7 months after local recurrence diagnosis. The cumulative 5-year local

Table 2. Treatment characteristics $(n=133)$

\begin{tabular}{lc}
\hline \multicolumn{1}{c}{ Characteristic } & No. of patients (\%) \\
\hline Types of axillary management & \\
Axillary lymph node dissection & $110(82.7)$ \\
Sentinel lymph node biopsy & $23(17.3)$ \\
Chemotherapy & \\
AC followed by T & $95(71.4)$ \\
AC & $9(6.8)$ \\
CMF & $10(7.5)$ \\
TC & $7(5.3)$ \\
Other & $3(2.3)$ \\
No & $9(6.8)$ \\
Anti-hormonal therapy & \\
Tamoxifen & $75(56.4)$ \\
Aromatase inhibitor & $37(27.8)$ \\
No & $21(15.8)$ \\
Trastuzumab & \\
Yes & $33(24.8)$ \\
No & $100(75.2)$
\end{tabular}

AC, adriamycin and cyclophosphamide; $T$, taxane; CMF, cyclophosphamide, methotrexate, and fluorouracil; TC, taxane and cyclophosphamide. 
A

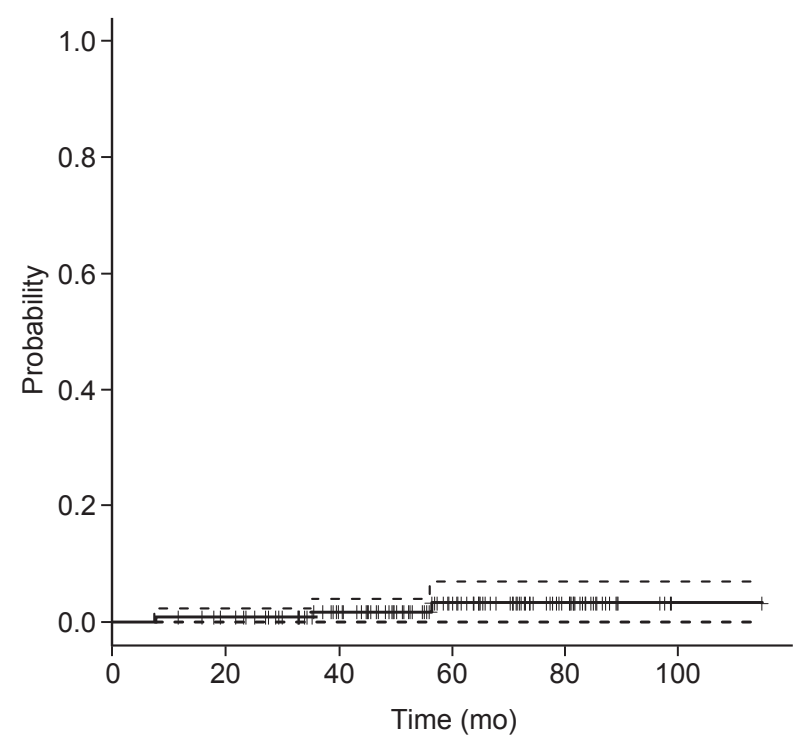

C

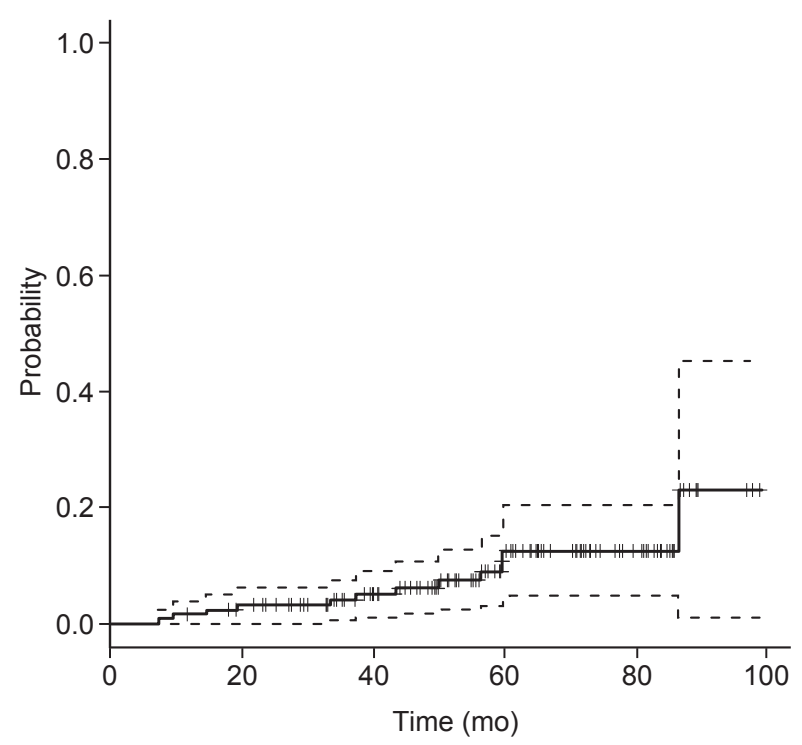

B

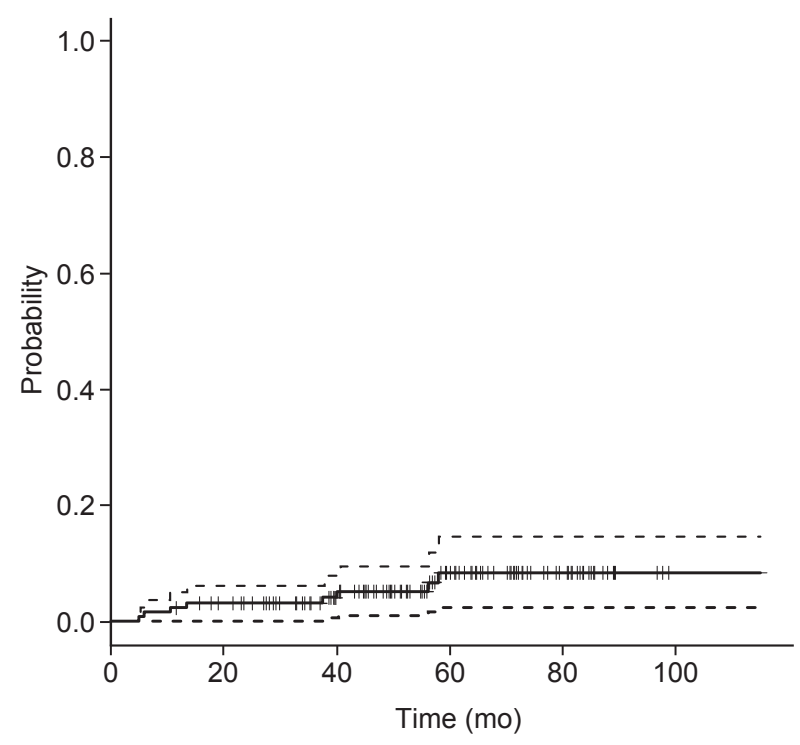

D

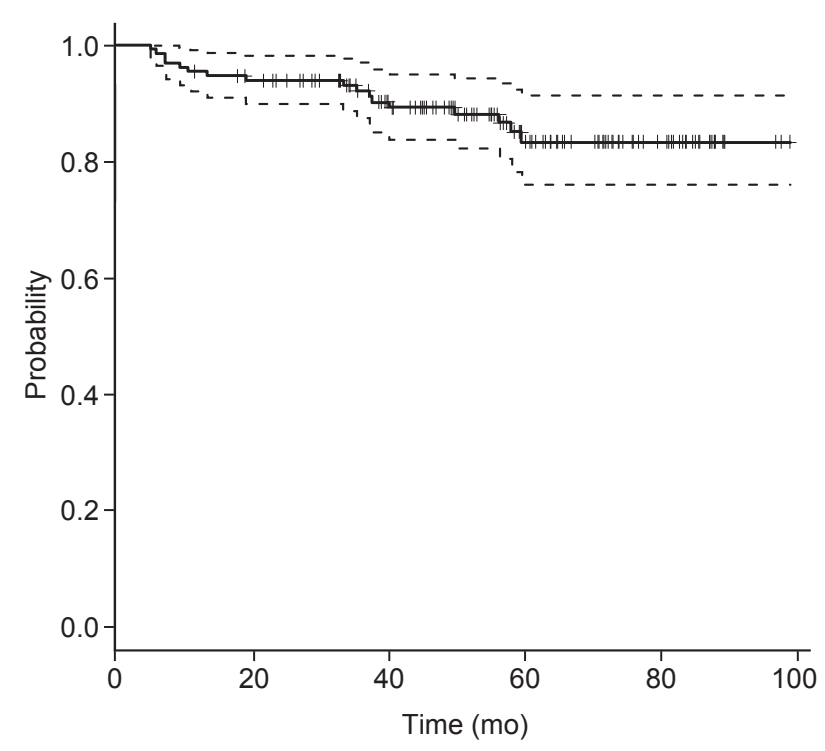

Fig. 1. Kaplan-Meier curves for local recurrence (A), regional recurrence (B), distant metastasis (C), and disease-free survival (D) for all 133 study patients.

recurrence rate was 3.1\% (Fig. 1). There were no statistically significant clinicopathologic factors associated with local recurrence.

Eight patients (6.0\%) experienced regional recurrence (Table 3). The median time to regional recurrence was 26 months (range, 5 to 58 months). Seven patients had isolated regional recurrence, and 1 patient was diagnosed with local recurrence and distant metastasis concurrently with regional recurrence.
Three patients with regional recurrence later developed distant metastases at 6,24 , and 46 months after the diagnosis of regional recurrence. The cumulative 5 -year regional recurrence rate was $8.0 \%$. Among the clinical and pathologic factors, lymphovascular invasion $(p=0.015)$ was associated with an increased risk of regional recurrence (univariate $p=0.015$ and multivariate $p=0.054$; hazard ratio $(H R)=7.91 ; 95 \%$ confidence interval (CI), 0.96-64.97) (Table 4, Fig. 2). Of note, 


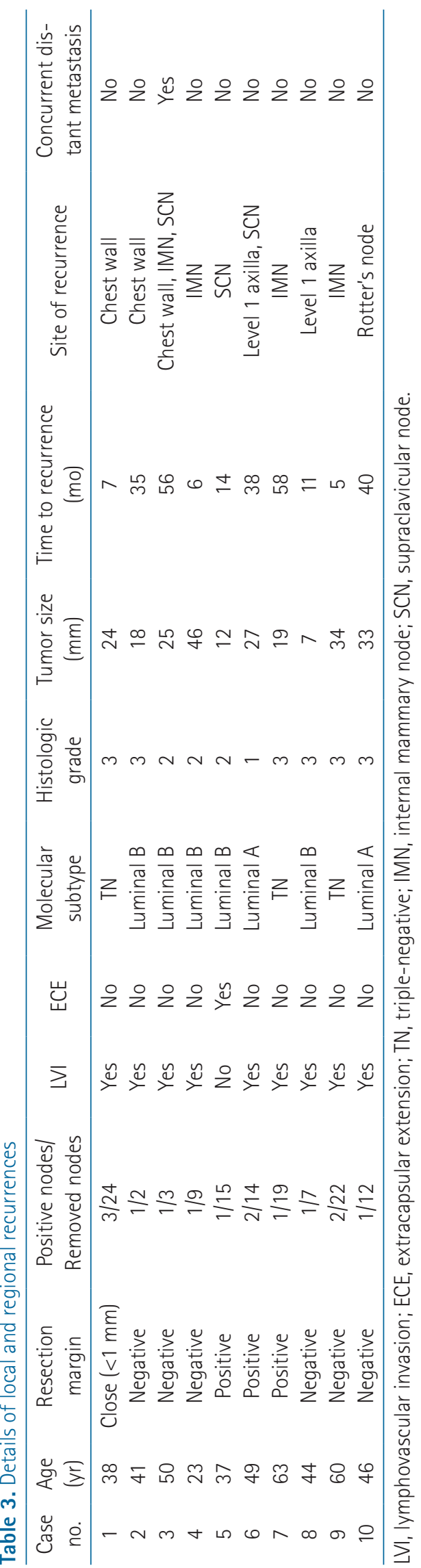

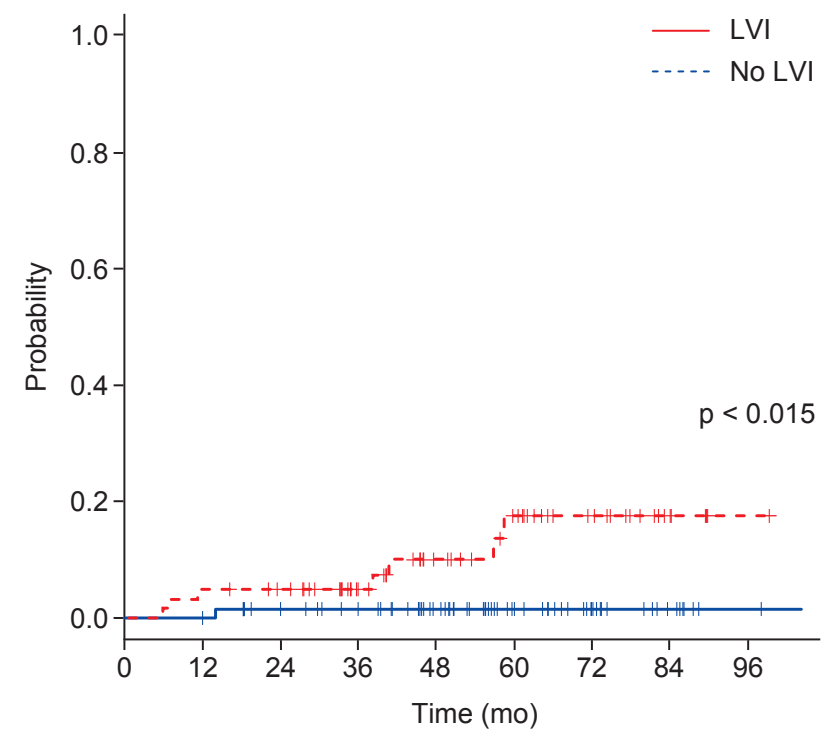

Fig. 2. Kaplan-Meier curves for regional recurrence for patients with lymphovascular invasion (LVI, dashed red line) and without LVI (blue line).

patients with lymphovascular invasion were at considerable risk for 5-year recurrence (16.1\%).

\section{Distant metastasis and disease-free survival}

Twelve patients (9.0\%) had a distant metastasis. Of these, isolated distant metastasis developed in 7 patients. Median time to distant metastasis was 69.5 months (range, 16 to 115 months). The site of distant metastasis was bone in 6 patients, lung in 4 , liver in 1 , and liver and bone in 1 patient.

The cumulative 5-year distant metastasis and diseasefree survival rate were $11.7 \%$ and $83.4 \%$, respectively. On univariate analysis, factors associated with an increased distant metastasis risk were non-invasive ductal carcinoma (IDC) pathology $(p<0.001)$, ER-negative tumor ( $p=0.003)$, and PR-negative tumor $(p=0.034)$. Among these factors, non-IDC pathology ( $\mathrm{HR}=1.86 ; 95 \% \mathrm{Cl}, 1.18-2.94 ; \mathrm{p}=0.008)$ and estrogen receptor-negative tumor $(\mathrm{HR}=0.16 ; 95 \% \mathrm{Cl}$, $0.05-0.52 ; p=0.003$ ) remained significant in the multivariate analysis.

Regarding disease-free survival, non-IDC pathology $(p=$ $0.002)$, histologic grade $3(p=0.004)$, ER-negative tumor ( $p$ $=0.008)$, and PR-negative tumor $(p=0.045)$ were associated with worse outcomes in univariate analysis. Among these factors, non-IDC pathology $(\mathrm{HR}=2.91 ; 95 \% \mathrm{Cl}, 1.65-5.14 ; \mathrm{p}$ $<0.001)$ was significantly associated with poorer disease-free survival on multivariate analysis. 
Table 4. Regional recurrence with respect to clinicopathologic factors

\begin{tabular}{|c|c|c|c|}
\hline & \multirow{2}{*}{$\begin{array}{l}5 \text {-yr risk of regional } \\
\text { recurrence }(\%)\end{array}$} & \multicolumn{2}{|c|}{$p$-value } \\
\hline & & Univariate & Multivariate $(\mathrm{HR}, 95 \% \mathrm{Cl})$ \\
\hline Age (yr) & & 0.691 & \\
\hline$\leq 40$ & 8.0 & & \\
\hline$>40$ & 8.2 & & \\
\hline Pathology & & 0.796 & \\
\hline IDC & 8.1 & & \\
\hline Non-IDC & 7.1 & & \\
\hline Tumor size $(\mathrm{cm})$ & & 0.840 & \\
\hline$\leq 2$ & 7.8 & & \\
\hline$>2$ & 8.3 & & \\
\hline Histologic grade & & 0.098 & $0.332(1.80,0.55-5.89)$ \\
\hline $1-2$ & 5.3 & & \\
\hline 3 & 16.0 & & \\
\hline Nuclear grade & & 0.513 & \\
\hline $1-2$ & 0.0 & & \\
\hline 3 & 8.6 & & \\
\hline Multiple & & 0.153 & \\
\hline Yes & 15.2 & & \\
\hline No & 5.4 & & \\
\hline Estrogen receptor & & 0.848 & \\
\hline Positive & 7.3 & & \\
\hline Negative & 10.0 & & \\
\hline Progesterone receptor & & 0.981 & \\
\hline Positive & 8.0 & & \\
\hline Negative & 8.0 & & \\
\hline HER2 & & 0.279 & \\
\hline Positive & 2.9 & & \\
\hline Negative & 10.7 & & \\
\hline Ki-67 (\%) & & 0.166 & \\
\hline$<14$ & 4.1 & & \\
\hline$\geq 14$ & 10.9 & & \\
\hline Molecular subtype & & 0.247 & \\
\hline Luminal A & 4.3 & & \\
\hline Non-luminal A & 10.6 & & \\
\hline Lymphovascular invasion & & 0.015 & $0.054(7.91,0.96-64.97)$ \\
\hline Yes & 16.1 & & \\
\hline No & 1.5 & & \\
\hline Extracapsular extension & & 0.236 & \\
\hline Yes & 16.7 & & \\
\hline No & 7.7 & & \\
\hline Positive lymph node & & 0.078 & \\
\hline 1 & 14.3 & & \\
\hline$\geq 2$ & 3.1 & & \\
\hline Nodal ratio ${ }^{a)}(\%)$ & & 0.250 & \\
\hline$<20$ & 9.4 & & \\
\hline$\geq 20$ & 4.5 & & \\
\hline
\end{tabular}

IDC, invasive ductal carcinoma; HER2, human epidermal growth factor receptor 2 ; $\mathrm{HR}$, hazard ratio; $\mathrm{Cl}$, confidence interval.

a) Ratio of positive lymph nodes of the total dissected lymph nodes. 


\section{Discussion and Conclusion}

In this study, 5-year local and regional recurrence risk were 3.1\% and $8.0 \%$, respectively, for breast cancer patients with tumors $<5 \mathrm{~cm}$ and 1-3 positive lymph nodes who were treated with mastectomy and modern adjuvant systemic therapy and who did not receive PMRT. Our results are consistent with recent reports that implemented modern systemic therapy [7-10].

Historical trials have shown higher locoregional recurrence rates in breast cancer patients with 1-3 positive nodes who did not receive PMRT, compared to those reported from modern trials. Specifically, the Danish 82B/C trials [3] showed a 15year locoregional recurrence risk of $27 \%$ and the Vancouver British Columbia trial [2] noted a 20-year isolated locoregional recurrence risk of $21 \%$. PMRT was associated with a significant improvement in both locoregional control and survival for both trials $[2,3]$. Furthermore, the EBCTCG meta-analysis of data from 22 randomized trials revealed that radiotherapy reduced locoregional recurrence and breast cancer mortality in patients with 1-3 positive nodes [5]. Based on these findings, PMRT was recommended for these patients despite the possibility of radiation-related toxicity.

The standard systemic treatment of breast cancer has recently changed. Most patients in the aforementioned studies were administered adjuvant chemotherapy with cyclophosphamide, methotrexate, and 5-fluorouracil (CMF regimen); in that era, trastuzumab had not yet been introduced. Anthracycline- and taxane-based chemotherapy has been shown to be more effective than the CMF regimen, and has become the standard adjuvant chemotherapy for node-positive breast cancer patients [14-16]. In addition, the introduction of trastuzumab and the routine use of tamoxifen or aromatase inhibitors for hormonal receptor-positive disease could further reduce breast cancer recurrence and mortality. Results from the Eastern Cooperative Oncology Group [17] and National Surgical Adjuvant Breast and Bowel Project [18] trials found a locoregional recurrence rate of 13\%. Likewise, in a study by McBride et al. [7], the locoregional recurrence rate was lower during the late era than during the early era. Although radiotherapy reduced locoregional recurrence in the early era cohort, it did not benefit patients treated in the later cohort who were also treated with modern systemic treatments. In contrast, a similar study from Korea reported improved disease-free survival in patients treated with PMRT compared to those without radiotherapy during the late era [19]. Additionally, they reported an increased risk of distant metastasis without PMRT in patients in the late era.
Accordingly, various studies showed conflicting results regarding the clinical benefit of PMRT in patients with 1-3 positive node in the modern treatment era. Nevertheless, it seems clear that the absolute locoregional recurrence risk has decreased over time in patients treated with modern systemic treatment, and subsequently, the absolute benefit of PMRT is lower when compared with past experience. Similar to other recent studies [7-10,20], our results showed a respective 5 -year local recurrence and regional recurrence risk of 3.1\% and $8.0 \%$, which was lower than that of the Danish and British Columbia trials.

The reduced locoregional recurrence risk in node-positive patients led to the attempted selection of high-risk patients for locoregional recurrence, indicated by a sufficient risk of microscopic residual disease [8,20-24]. Katz et al. [21] suggested that patients with $\geq 20 \%$ of involved nodes or $<20 \%$ involved nodes and a tumor size $\geq 5 \mathrm{~cm}$ have significant locoregional recurrence risk and would benefit from PMRT. Lai et al. [8] showed that age $\leq 40$ years, tumor size $\geq 3 \mathrm{~cm}$, and the presence of extensive intraductal components were risk factors for locoregional recurrence, and a 5-year locoregional recurrence risk of $25 \%$ was found in patients with 2 or more risk factors. In a study by Truong et al. [22], age $<45$ years, tumor size $>2 \mathrm{~cm}$, histologic grade 3, ER-negative disease, medial tumor location, $>1$ positive node, $>25 \%$ of nodes being positive were risk factors for recurrence-free survival. Another study analyzed supraclavicular nodal failure in 1-3 nodepositive breast cancer patients revealed that lymphovascular invasion, extracapsular extension, the number of involved nodes, and the level of involved nodes were prognostic factors [23]. Similarly, a retrospective multicenter analysis from Korea [20] also found histologic grade 3 as a risk factor for locoregional recurrence. In our study, lymphovascular invasion was a risk factor for regional recurrence, and patients with lymphovascular invasion had a 5-year regional recurrence rate of $16.1 \%$. Taken together, these findings suggest that patients with such poor prognostic factors (e.g., high histologic grade or Iymphovascular invasion) might benefit from PMRT to the regional lymphatic area.

Previous studies were primarily concerned about whom to treat, and they did not consider how to treat breast cancer patients with 1-3 positive nodes. PMRT fields usually include the chest wall and regional nodal region (supraclavicular region, infraclavicular region, internal mammary nodes, and any part of the axillary region) [6]. In our study, among the 10 patients with locoregional recurrence, only 3 had chest wall recurrence (2 with isolated chest wall recurrence and 
1 with both chest wall and lymph node recurrence). The cumulative 5 -year local recurrence risk was only 3.1\%. Failure patterns found in a study of Lai et al. [8] were similar to our results, in that they noted that regional recurrence was a more frequent failure pattern in breast cancer patients with 1-3 positive nodes who were treated with mastectomy and adjuvant systemic treatment. Likewise, a Korean multicenter study (KROG 14-18) also reported that the frequency of local recurrence (6/584 patients) had an incidence of approximately 1/4 for regional recurrence (22/584 patients) [20]. Other recently published results from two Korean multicenter studies (KROG 14-22 and KROG 14-23) showed very low chest wall recurrence rate in patients with $<5 \mathrm{~cm}$ tumor and 1-3 positive lymph nodes [25]. Based on these results, the authors questioned the necessity of chest wall radiotherapy in this group of patients.

Various complications may occur following chest wall irradiation. Frequent symptoms include chest wall pain, acute dermatitis, and late skin fibrosis. In addition, for leftsided breast cancer patients, an increase in cardiac-related mortality has been observed [26,27]. Specifically, Darby et al. [28] reported that the rate of major coronary events increased linearly with the heart mean dose by $7.4 \%$ per Gy. Furthermore, high rates of late contraction, fibrosis, capsular contracture, reconstructive failure, and poor cosmetic outcome were reported for patients who received immediate breast reconstruction followed by PMRT. Complications of reconstructed breast more frequently occur for patients who had implant reconstruction, compared with those that had autologous reconstruction $[10,29]$. In a recent prospective study on breast reconstruction by the Mastectomy Reconstruction Outcomes Consortium (MROC), PMRT was associated with an increased risk of complications at 2 years for patients who had an implant reconstruction, while PMRT had similar risks in patients who received autologous reconstruction [30]. Given the rising expectations of cosmetic outcomes in breast cancer patients in the contemporary era, PMRT needs to be more sophisticated and tailored. In this context, the local recurrence risk might need to be considered separately from the regional recurrence risk. The low risk of local recurrence shown in our study and in other studies suggests the possibility of irradiating the regional nodal area and omitting the chest wall for patients who have a high risk for regional recurrence (Fig. 3). In doing so, complications

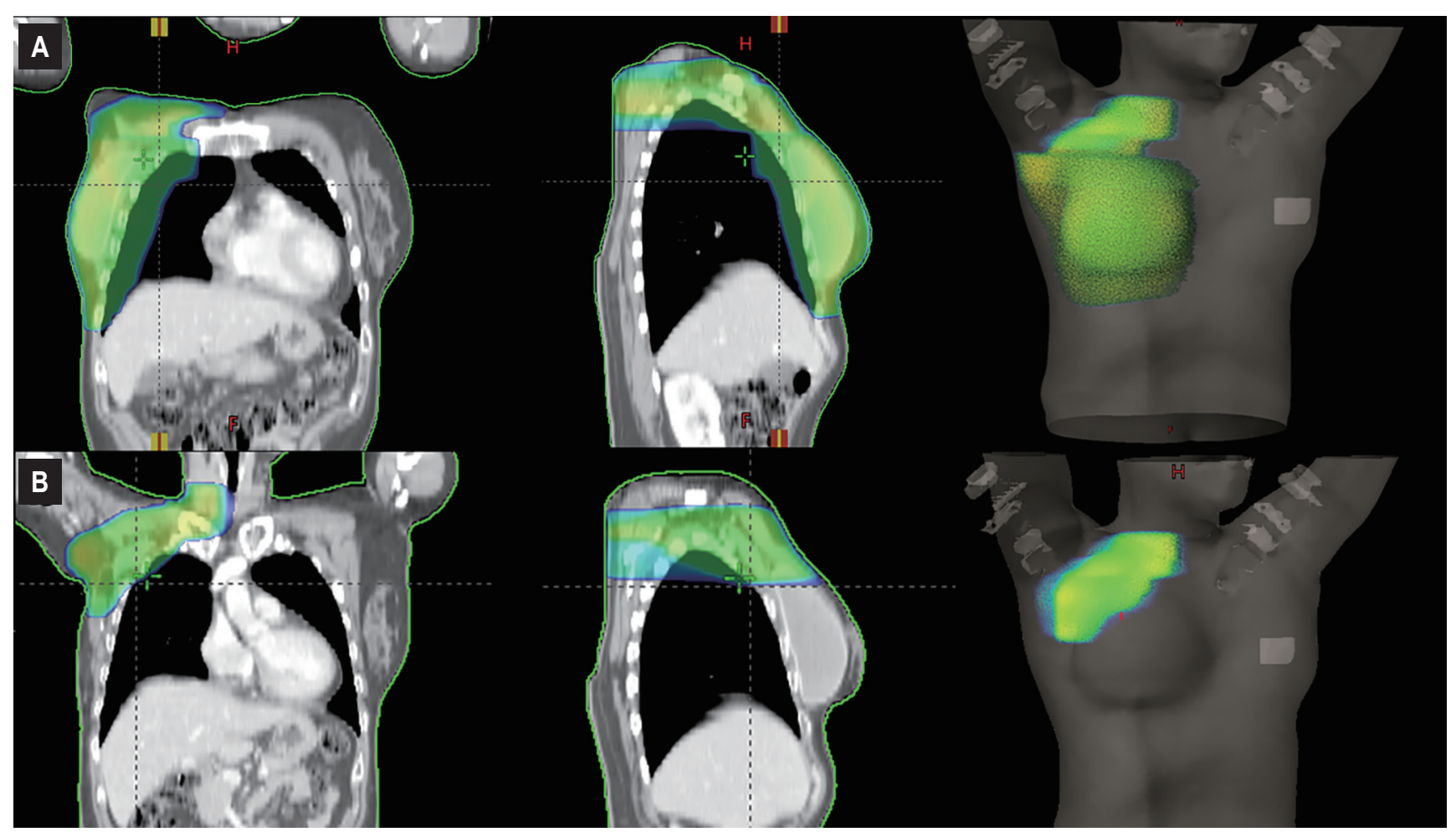

Fig. 3. Postmastectomy radiotherapy treatment plans for a 53-year-old female with breast cancer who underwent mastectomy, sentinel node biopsy, and immediate implant reconstruction. Radiotherapy volume included the ipsilateral chest wall, axillary, and supraclavicular region (A); while only the axillary and supraclavicular region without covering the ipsilateral chest wall (B). 
related to chest wall irradiation could be alleviated. However, the current known risk factors from most previous studies concerned locoregional recurrence as a single entity. Hence, to safely omit chest wall irradiation from PMRT for patients with a high risk of regional recurrence, further data should be collected.

Our study has some limitations. First, there may be unknown selection bias due to the retrospective design. In particular, many patients were treated with neoadjuvant chemotherapy since 2008 and there is a possibility that we included lowrisk, node-positive patients. Second, the total number of patients and the number of recurrence events may be too small to ensure statistical relevance of our results. Third, a relatively short follow-up period also limits our study findings. Nevertheless, our results provide information regarding local and regional recurrence separately, which may be used to support radiotherapy that is tailored according to whether risk of recurrence is local or regional. Our results warrant further investigation in future trials.

In conclusion, our study showed a very low 5-year local recurrence rate of $3.1 \%$ in breast cancer patients with a tumor size of $<5 \mathrm{~cm}$ and 1-3 positive nodes who were treated with mastectomy and modern adjuvant systemic treatment without radiotherapy. No significant risk factors associated with increased local recurrence were found. The PMRT volume need to be tailored for each patient's given risk for local and regional recurrence, considering the possible toxicities and benefits of chest wall irradiation.

\section{Conflict of Interest}

No potential conflict of interest relevant to this article was reported.

\section{References}

1. Ragaz J, Jackson SM, Le $N$, et al. Adjuvant radiotherapy and chemotherapy in node-positive premenopausal women with breast cancer. N Engl J Med 1997;337:956-62.

2. Ragaz J, Olivotto IA, Spinelli JJ, et al. Locoregional radiation therapy in patients with high-risk breast cancer receiving adjuvant chemotherapy: 20-year results of the British Columbia randomized trial. J Natl Cancer Inst 2005;97:11626.

3. Overgaard M, Nielsen HM, Overgaard J. Is the benefit of postmastectomy irradiation limited to patients with four or more positive nodes, as recommended in international consensus reports? A subgroup analysis of the DBCG 82 betc randomized trials. Radiother Oncol 2007;82:247-53.

4. Ceilley $E_{1}$ Jagsi $R$, Goldberg $S$, et al. Radiotherapy for invasive breast cancer in North America and Europe: results of a survey. Int J Radiat Oncol Biol Phys 2005;61:365-73.

5. EBCTCG (Early Breast Cancer Trialists' Collaborative Group). Effect of radiotherapy after mastectomy and axillary surgery on 10-year recurrence and 20-year breast cancer mortality: meta-analysis of individual patient data for 8135 women in 22 randomised trials. Lancet 2014;383:2127-35.

6. National Comprehensive Cancer Network. NCCN Clinical Practice Guidelines in Oncology: breast cancer (Version 1.2018) [Internet]. Plymouth Meeting, PA: National Comprehensive Cancer Network; c2018 [cited 2018 Nov 26]. Available from: https://www.nccn.org/professionals/physician_gls/default. aspx.

7. McBride A, Allen P, Woodward W, et al. Locoregional recurrence risk for patients with $T 1,2$ breast cancer with 1-3 positive lymph nodes treated with mastectomy and systemic treatment. Int J Radiat Oncol Biol Phys 2014;89:392-8.

8. Lai SF, Chen YH, Kuo WH, et al. Locoregional recurrence risk for postmastectomy breast cancer patients with T1-2 and one to three positive lymph nodes receiving modern systemic treatment without radiotherapy. Ann Surg Oncol 2016;23:3860-9.

9. Sharma R, Bedrosian I, Lucci A, et al. Present-day locoregional control in patients with $\mathrm{T} 1$ or $\mathrm{T} 2$ breast cancer with 0 and 1 to 3 positive lymph nodes after mastectomy without radiotherapy. Ann Surg Oncol 2010;17:2899-908.

10. Tendulkar RD, Rehman S, Shukla ME, et al. Impact of postmastectomy radiation on locoregional recurrence in breast cancer patients with 1-3 positive lymph nodes treated with modern systemic therapy. Int J Radiat Oncol Biol Phys 2012;83:e577-81.

11. Reisenbichler ES, Lester SC, Richardson AL, Dillon DA, Ly $A$, Brock JE. Interobserver concordance in implementing the 2010 ASCO/CAP recommendations for reporting ER in breast carcinomas: a demonstration of the difficulties of consistently reporting low levels of ER expression by manual quantification. Am J Clin Pathol 2013;140:487-94.

12. Rakha EA, Starczynski J, Lee AH, Ellis IO. The updated ASCO/ CAP guideline recommendations for HER2 testing in the management of invasive breast cancer: a critical review of their implications for routine practice. Histopathology 2014;64:609-15.

13. Goldhirsch A, Wood WC, Coates AS, et al. Strategies for subtypes: dealing with the diversity of breast cancer: 
highlights of the St. Gallen International Expert Consensus on the Primary Therapy of Early Breast Cancer 2011. Ann Oncol 2011;22:1736-47.

14. Mamounas EP, Bryant J, Lembersky B, et al. Paclitaxel after doxorubicin plus cyclophosphamide as adjuvant chemotherapy for node-positive breast cancer: results from NSABP B-28. J Clin Oncol 2005;23:3686-96.

15. Sartor $\mathrm{Cl}$, Peterson $\mathrm{BL}$, Woolf $\mathrm{S}$, et al. Effect of addition of adjuvant paclitaxel on radiotherapy delivery and locoregional control of node-positive breast cancer: cancer and leukemia group B 9344. J Clin Oncol 2005;23:30-40.

16. Early Breast Cancer Trialists' Collaborative Group (EBCTCG). Comparisons between different polychemotherapy regimens for early breast cancer: meta-analyses of long-term outcome among 100,000 women in 123 randomised trials. Lancet 2012;379:432-44.

17. Recht $A$, Gray $R$, Davidson $N E$, et al. Locoregional failure 10 years after mastectomy and adjuvant chemotherapy with or without tamoxifen without irradiation: experience of the Eastern Cooperative Oncology Group. J Clin Oncol 1999;17:1689-700.

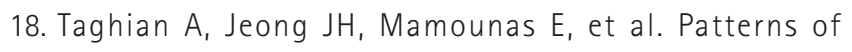
locoregional failure in patients with operable breast cancer treated by mastectomy and adjuvant chemotherapy with or without tamoxifen and without radiotherapy: results from five National Surgical Adjuvant Breast and Bowel Project randomized clinical trials. J Clin Oncol 2004;22:4247-54.

19. Chang JS, Lee J, Kim KH, et al. Do recent advances in diagnostic and therapeutic procedures negate the benefit of postmastectomy radiotherapy in $\mathrm{N} 1$ patients with a low risk of locoregional recurrence? Medicine (Baltimore) 2015;94:e1259.

20. Kim YJ, Park W, Ha B, et al. Postmastectomy radiotherapy in patients with $\mathrm{pT} 1-2 \mathrm{~N} 1$ breast cancer treated with taxanebased chemotherapy: a retrospective multicenter analysis (KROG 1418). Cancer Res Treat 2017;49:927-36.

21. Katz $A$, Buchholz TA, Thames $H$, et al. Recursive partitioning analysis of locoregional recurrence patterns following mastectomy: implications for adjuvant irradiation. Int J Radiat Oncol Biol Phys 2001;50:397-403.

22. Truong PT, Olivotto IA, Kader HA, Panades $M$, Speers $C H$, Berthelet E. Selecting breast cancer patients with T1-T2 tumors and one to three positive axillary nodes at high postmastectomy locoregional recurrence risk for adjuvant radiotherapy. Int J Radiat Oncol Biol Phys 2005;61:1337-47.

23. Yu JI, Park W, Huh SJ, et al. Determining which patients require irradiation of the supraclavicular nodal area after surgery for N1 breast cancer. Int J Radiat Oncol Biol Phys 2010;78:113541.

24. Wen G, Zhang JS, Zhang YJ, Zhu YJ, Huang XB, Guan XX. Predictive value of molecular subtyping for locoregional recurrence in early-stage breast cancer with $\mathrm{N} 1$ without postmastectomy radiotherapy. J Breast Cancer 2016;19:17684.

25. Chang JH, Shin KH, Ahn SD, et al. Chest wall recurrence in pT1-2N0-1 breast cancer patients after mastectomy without radiotherapy. Breast Cancer Res Treat 2018;169:507-12.

26. Darby SC, McGale P, Taylor CW, Peto R. Long-term mortality from heart disease and lung cancer after radiotherapy for early breast cancer: prospective cohort study of about 300,000 women in US SEER cancer registries. Lancet Oncol 2005;6:557-65.

27. Paszat LF, Mackillop WJ, Groome PA, Schulze K, Holowaty E. Mortality from myocardial infarction following postlumpectomy radiotherapy for breast cancer: a populationbased study in Ontario, Canada. Int J Radiat Oncol Biol Phys 1999:43:755-62.

28. Darby SC, Ewertz M, McGale $P$, et al. Risk of ischemic heart disease in women after radiotherapy for breast cancer. $N$ Eng J Med 2013;368:987-98.

29. Barry M, Kell MR. Radiotherapy and breast reconstruction: a meta-analysis. Breast Cancer Res Treat 2011;127:15-22.

30. Jagsi R, Momoh AO, Qi J, et al. Impact of radiotherapy on complications and patient-reported outcomes after breast reconstruction. J Natl Cancer Inst 2018;110:157-65. 